Bioimpedance of soft tissue under compression

This article has been downloaded from IOPscience. Please scroll down to see the full text article.

2012 Physiol. Meas. 331095

(http://iopscience.iop.org/0967-3334/33/6/1095)

View the table of contents for this issue, or go to the journal homepage for more

Download details:

IP Address: 141.211.173.82

The article was downloaded on 26/06/2013 at 15:16

Please note that terms and conditions apply. 


\title{
Bioimpedance of soft tissue under compression
}

\author{
R E Dodde, J L Bull and A J Shih \\ Biomedical Engineering Department, University of Michigan, Ann Arbor, MI, USA \\ E-mail: dodderob@umich.edu
}

Received 13 December 2011, accepted for publication 24 April 2012

Published 24 May 2012

Online at stacks.iop.org/PM/33/1095

\begin{abstract}
In this paper compression-dependent bioimpedance measurements of porcine spleen tissue are presented. Using a Cole-Cole model, nonlinear compositional changes in extracellular and intracellular makeup; related to a loss of fluid from the tissue, are identified during compression. Bioimpedance measurements were made using a custom tetrapolar probe and bioimpedance circuitry. As the tissue is increasingly compressed up to $50 \%$, both intracellular and extracellular resistances increase while bulk membrane capacitance decreases. Increasing compression to $80 \%$ results in an increase in intracellular resistance and bulk membrane capacitance while extracellular resistance decreases. Tissues compressed incrementally to $80 \%$ show a decreased extracellular resistance of $32 \%$, an increased intracellular resistance of $107 \%$, and an increased bulk membrane capacitance of $64 \%$ compared to their uncompressed values. Intracellular resistance exhibits double asymptotic curves when plotted against the peak tissue pressure during compression, possibly indicating two distinct phases of mechanical change in the tissue during compression. Based on these findings, differing theories as to what is happening at a cellular level during high tissue compression are discussed, including the possibility of cell rupture and mass exudation of cellular material.
\end{abstract}

Keywords: bioimpedance, spleen, soft tissue, compression, strain

(Some figures may appear in colour only in the online journal)

\section{Introduction}

Tissue undergoes compression in a variety of physiological, clinical, and research experiences and on a large range of scales. Physiological examples include the result of pressure exerted on vessel walls during the cardiac cycle (Fung 1993), blunt trauma and traumatic brain injuries (Eaton 2005), and at the cellular level mechanotransduction (Demou 2010, Ingber 2006). Clinical and research examples are very diverse, including edema monitoring (pitting edema) in dialysis patients (Berry et al 2008), the use of electrosurgical forceps while coagulating tissue (Dodde et al 2008), and optical clearing techniques for increasing the depth of field using optical imaging techniques (Rylander et al 2006). 
Much research has been performed on the mechanical response of soft tissue under compression (Tamura et al 2002, Rosen et al 2008, Abramowitch 2004). Depending on the tissue being tested, various models have been used to explain the measured phenomenon using viscoelastic, poroelastic, and biphasic theories (Mow et al 1980, Fung 1993, Pena et al 1998). However, it proves difficult to relate the mathematical parameters to the physiological consequences during tissue strain.

Additionally, little within the literature has been published regarding material property changes tissue experiences while undergoing compression. It is generally assumed that among these changes is a loss of fluid from the tissue space (Rylander et al 2006). More specifically, the fluid loss has been postulated to come primarily from the extracellular compartments of the compressed tissue (González-Correa 2005). In theory, bioimpedance techniques can distinguish between intracellular fluid, extracellular fluid, and the bulk capacitance of the cellular membranes and thus offer a methodology for a more complete understanding of the changes occurring within tissue during compression.

Previous literature has noticed a change in bioimpedance readings with an increase in pressure applied to measurement devices. González-Correa et al (González-Correa 2005) showed that for various tissues the resistivity increases as pressure is increased to the measurement probe. Keshtkar et al (Keshtkar 2008) showed that independent of pressure readings, the ratio of probe size to effective electrode size must be considered as well. Larger probes tend to report lower impedance readings than their smaller counterparts given a constant pressure and electrode size.

In both cases it is asserted that the loss of extracellular fluid is responsible for the increase in electrical impedance readings; however, only the real part of the impedance is shown. Without knowledge of the complex impedance, including the imaginary component, it cannot be determined if it is a loss of interstitial fluid, intracellular fluid, or a combination of both.

The goal of this research is to identify the effect tissue compression has on bioimpedance readings to gain insight into the intracellular and interstitial fluid distribution in tissue. The standard Cole-Cole model (Cole and Cole 1941) is extended to incorporate strain-dependent values is suggested for analysis of compressed tissue due to the changes in both the loci diameter and position along the real axis. As the components of the Cole-Cole model can be mapped to the intracellular and extracellular fluid compartments of tissue, a hypothesis for what happens to the tissue's cellular material during compression is discussed based on comparisons to peak pressures developed within the tissue during incremental tissue compression.

\section{Materials and methods}

For this study, bioimpedance measurements were collected using a standard tetrapolar technique (Bouty 1884, Schwan 1968b, Jakosky 1961, Ackmann 1993) on porcine spleen under incremental compressive strains once the tissue was seen to relax from the initial compressive stress. Spleen tissue was chosen for its uniform thickness throughout the organ as well as its general isotropy (Bowdler 2002). Bioimpedance measurements were conditioned to remove circuitry effects and experimental stray capacitance before fitting the measurements to a Cole-Cole model. Details for the experimental setup, experimental method, data conditioning, and Cole-Cole fitting are given below.

\subsection{Experimental setup}

The bioimpedance probe uses four $0.25 \mathrm{~mm}$ diameter platinum electrodes and is $19 \mathrm{~mm}$ in diameter as shown in figure 1(a). The electrodes are equally spaced $3.0 \mathrm{~mm}$ apart resulting in a 


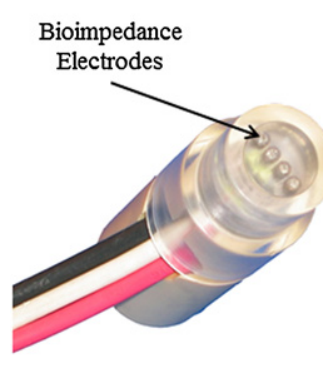

(a)

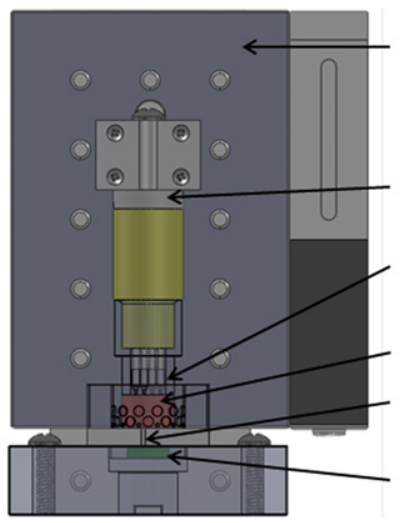

(b)

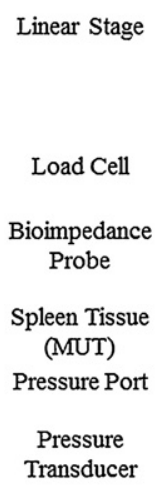

ransducer

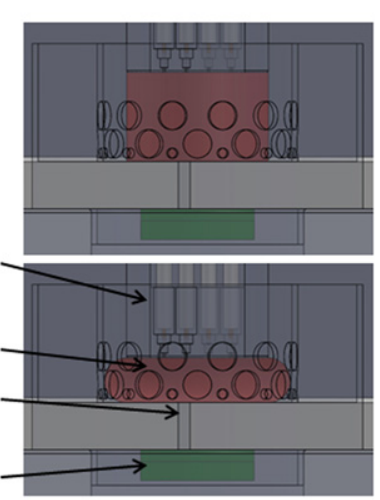

(c)

Figure 1. Overview of experimental setup. (a) Close-up view of the surface of the bioimpedance probe showing the four, equally spaced electrodes. (b) Schematic of entire experimental setup showing the linear stages, bioimpedance probe, spleen tissue, and location of the load cell and pressure transducers. (c) Close-up view of the bioimpedance probe and the spleen tissue under no compression (top) and 50\% compression (bottom).

geometric probe constant $(k)$ of $53 \mathrm{~m}^{-1}$ (Littwitz et al 1990). This electrode spacing has been shown to yield optimum measurement sensitivity (Brown et al 2000, Grimnes and Martinsen 2007). A load cell (LC8125, Omega, Stamford, CT) is placed in series with the bioimpedance probe and both are attached to a Siskiyou 200cri-R linear stage with $2.0 \mu \mathrm{m}$ positioning accuracy as shown in figure 1(b). The linear stage was used to drive the bioimpedance probe to compress the tissue. A custom-machined cylindrical acrylic chamber with a $25.4 \mathrm{~mm}$ diameter central cavity is used to hold the tissue as shown in figure 1(b). An array of radial holes (3.8 $\mathrm{mm}$ in diameter) through the sides of the chamber allow for the free flow of fluid out of the tissue during testing. A pressure sensor (NPC-1210, GE Measurement \& Control Solutions, Billerica, MA) is incorporated into the bottom of the tissue chamber and coupled to the tissue sample through a $3.0 \mathrm{~mm}$ diameter channel through the bottom of the tissue chamber.

Cylindrical porcine spleen tissue samples were punched out of whole porcine spleen using a sharpened $25.4 \mathrm{~mm}$ diameter leather punch. Samples were taken as far away from the midline as possible to avoid the large vessels located near the midline of the spleen.

Bioimpedance measurements were made using a custom circuit shown in figure 2. Current was injected into the tissue through an outer electrode (HC) using a voltage controlled current source consisting of a modified Howland current pump. The current source was designed to generate a $60 \mu \mathrm{A}$ root-mean-square (RMS) current signal to ensure the current density does not exceed levels known to heat tissue (Littwitz et al 1990). An integrator circuit was used to provide a dc feedback signal to minimize the dc offset and a series capacitor was used to further ac couple the signal to the tissue sample. Current exited out the opposite electrode (LC) and a current-to-voltage converter circuit in a transimpedance configuration was used to generate a signal proportional to the actual current with a gain of $20000 \mathrm{~V} \mathrm{~A}^{-1}$. An instrumentation amplifier (INA111, Texas Instruments, Dallas, TX) was used to generate a signal proportional to the voltage drop between the middle two electrodes (HV and LV) with a gain of $10 \mathrm{~V} \mathrm{~V}^{-1}$ and an additional gain of $2 \mathrm{~V} \mathrm{~V}^{-1}$ by passing the output from the instrumentation amplifier through an inverting amplifier. The inputs to the instrumentation amplifier were first buffered using voltage follower circuits with input resistance of $1 \mathrm{~T} \Omega$ to minimize current leaking out of the 


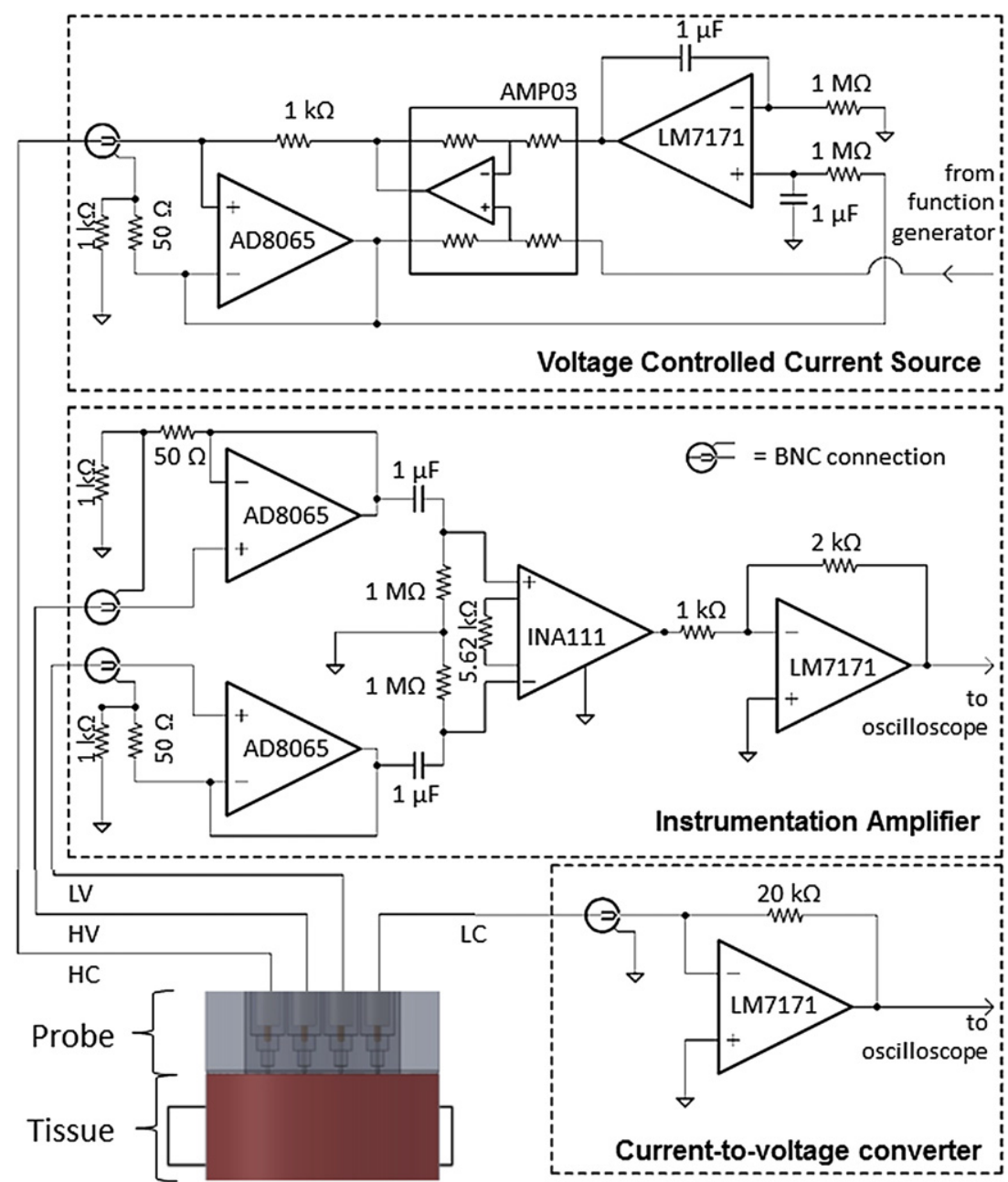

Figure 2. Schematic of the circuit developed to measure soft tissue bioimpedance using the tetrapolar method. The three main components of the circuit are a voltage controlled current source which injects current into the tissue through the signal line $\mathrm{HC}$, an instrumentation amplifier to measure differential voltage signals in the tissue measured at $\mathrm{HV}$ and $\mathrm{HC}$, and a current-to-voltage converter to monitor the current passing through the tissue from signal LC. Note that the output of the current source into the tissue has an additional series capacitor used to further remove any dc offset in the signal.

tissue sample through the signal lines. Circuit characterization is discussed in section 2.3.1. The cable shields for the output of the current source and the input to the voltage buffers in the IA were driven by low impedance voltages held slightly lower than the signal line voltage by passing the driving signal through a voltage divider.

An Agilent 33120A function generator was used to generate the input signal to the circuit and a National Instruments PXI-5114 oscilloscope was used to record the output signals from the instrumentation amplifier and current-to-voltage converter circuits at 100 times the input signal frequency. Pressure and load measurements were collected at $100 \mathrm{~Hz}$ using a PXI-6221 DAQ card. A PXI-7340 motion controller was used to control the linear 
stage. A Labview program was used to control the instrumentation hardware and record the experimental measurements.

\subsection{Experimental procedure}

Eight porcine spleen samples were tested from three animals. For each trial, the bottom of the tissue chamber was determined by lowering the bioimpedance probe into the empty tissue chamber until a load is detected by the load cell. The tissue sample was placed inside the tissue chamber and the bioimpedance probe was lowered until continuity was measured across the outer electrodes indicating the top of the tissue. A $60 \mu \mathrm{A}_{\mathrm{RMS}}$ current at 13 frequencies from $100 \mathrm{~Hz}$ to $1 \mathrm{MHz}(100 \mathrm{~Hz}, 200 \mathrm{~Hz}, 500 \mathrm{~Hz}, 1 \mathrm{kHz}, 2 \mathrm{kHz}, 5 \mathrm{kHz}, 10 \mathrm{kHz}$, etc) was passed through the tissue and bioimpedance measurements were recorded using a PXI-5044 oscilloscope. Measurements at frequencies above $100 \mathrm{kHz}$ were used only to characterize stray capacitance in the experimental setup. The tissue was then compressed using the bioimpedance probe to a $10 \%$ strain level at a rate of $1.5 \mathrm{~mm} \mathrm{~s}^{-1}$ while recording the resultant pressure on the tissue. Once the tissue had relaxed (pressure measurement decreased less than $5 \mathrm{kPa} \mathrm{min}^{-1}$ ) additional bioimpedance measurements were recorded. This cycle of compressing the tissue, recording the resultant tissue stress, and taking bioimpedance measurements after the tissue has relaxed was repeated in $10 \%$ strain increments until an $80 \%$ strain level had been reached.

It is well known that the electrical conductivity of tissue exhibits a temperature dependence of approximately $2 \% /{ }^{\circ} \mathrm{C}$ (Schwan and Foster 1980). Both high current densities and high strain rates can potentially increase tissue temperature and influence impedance measurements. Current densities were maintained low enough to avoid temperature increases in the tissue (Littwitz et al 1990). Also, the linear stage velocity did not exceed $1.5 \mathrm{~mm} \mathrm{~s}^{-1}$ in order to maintain low strain rates and avoid unwanted development of thermal energy due to friction during compression. Bioimpedance measurements were taken after the tissue had reached a point near relaxation, allowing for any thermal energy generated during compression to dissipate.

\subsection{Data conditioning}

Various factors are known to influence bioimpedance measurements and should be removed before analysis is performed. For this study, the recorded data is conditioned to remove influences from the operational amplifiers in the circuitry, distance of the bioimpedance electrodes to the bottom of the tissue chamber, and stray capacitance.

2.3.1. Characterization of the bioimpedance circuitry. The output signal produced by a circuit can be influenced in unintended ways due to issues such as input capacitance, bandwidth, slew rate limitations, and input/output impedances of the operational amplifiers used in the circuit and must be accounted for (Wojslaw and Moustakas 1986).

To characterize the response of the current-to-voltage converter circuitry, a known current was generated by passing a voltage signal through a known load placed in series with the input to the circuit. The input signal was compared to the output signal for gain and phase shift to characterize the circuit.

To characterize the voltage-controlled current source circuit, the current output was passed through resistances in the range of interest $(100$ to $100 \mathrm{k} \Omega$ ) which were then placed in series with the current-to-voltage converter circuit. While the impedance of the tissue was not expected to be greater than $5 \mathrm{k} \Omega$, contact impedance at low frequencies must also be driven by the current source so a larger load is tested to ensure adequate capabilities of the current 
source at low frequencies. The measured output current versus frequency for each load was characterized.

To characterize the instrumentation amplifier circuitry, a differential voltage signal in the range of interest ( 3 to $300 \mathrm{mV}_{\mathrm{RMS}}$ ) was input into the front-end buffers using a function generator. Considering a current input of $60 \mu \mathrm{A}_{\mathrm{RMS}}$, this differential voltage range covers the differential voltage signal expected from impedances ranging from $50-5 \mathrm{k} \Omega$. The input signal was compared to the output signal for gain and phase shift to characterize the circuit. Any detected phase shift was subsequently subtracted from the measured phase shift when taking a bioimpedance measurement.

2.3.2. Characterization of the influence of bioimpedance probe proximity to the bottom of the tissue chamber. As the tissue is compressed, the bioimpedance probe gets closer to the bottom of the acrylic tissue chamber which has much higher impedance than the tissue. It has previously been shown that impedance measurements are dependent on the distance of the probe to other dissimilar materials, especially as the change in impedance between differing layers increases (Tsai 2000, Kottam 2004, Steendijk et al 1993).

To characterize the experimental setup for this influence, the impedances of three known saline solution concentrations $(0.9 \%, 0.125 \%$ and $0.01 \%)$ covering the region of interest for expected loads $(50-5 \mathrm{k} \Omega$ ) were measured as a function of $h$. In this study, $h$ is defined as the distance $(\mathrm{mm})$ between the bioimpedance probe electrodes and the bottom of the tissue chamber. No changes in the impedance magnitude $(|Z|)$ were noticed when increasing $h$ beyond $11 \mathrm{~mm}$, so all saline impedance data were normalized to the measurement made at $h=11 \mathrm{~mm}$, denoted as $|Z|^{*}$. The influence $h$ has on $|Z|$ during a tissue test is removed by dividing the measured $|Z|$ by the corresponding $|Z|$ at the same $h$ measured in the saline tests normalized to $|Z|^{*}$.

2.3.3. Characterization of Hook effect. Stray capacitance to ground is a known concern when performing bioimpedance measurements (Scharfetter 1998, Bolton et al 1998, McEwan et al 2007). Sources of stray capacitance include the coaxial cables connecting the bioimpedance circuit to the bioimpedance probe, traces on the bioimpedance circuit PCB board, and routes to ground through the acrylic tissue chamber. The methodology of Buendía et al (2010) for removing the effect of stray capacitance on bioimpedance measurements is used in this experiment. Due to the negligible susceptance $(\operatorname{Im}|Y|$, where $Y=1 / Z)$ of tissue at high frequencies, the stray capacitance $\left(C_{p}\right)$ is estimated by the slope of the graph of susceptance plotted against natural frequency $(\omega$, where $\omega=2 \pi f)$. Susceptance values from bioimpedance measurements taken at $200 \mathrm{kHz}, 500 \mathrm{kHz}$ and $1 \mathrm{MHz}$ were plotted against $\omega$ and a linear fit calculated to estimate $C_{p}$. Its influence on the measurement reading was removed using the following relationship:

$$
Z_{c}=Z_{m} \mathrm{e}^{-\log \left(1-\mathrm{j} \omega C_{p} Z_{m}\right)}
$$

where $Z_{c}$ is the conditioned impedance measurement after removing $C_{p}, Z_{m}$ is the measured impedance and $\mathrm{j}$ is $\sqrt{-1}$ (Buendía, et al 2010).

\subsection{Cole-Cole model parameter fitting}

The real part of the conditioned data was fit to a Cole-Cole model using a non-linear least squares fitting trust-region algorithm as discussed by Ayllon et al (2009):

$$
R(\omega)=R_{\infty}+\frac{\left(R_{0}-R_{\infty}\right)\left(1+\left(\frac{\omega}{\omega_{c}}\right)^{\alpha} \cos \left(\frac{\alpha \pi}{2}\right)\right)}{1+2\left(\frac{\omega}{\omega_{c}}\right)^{\alpha} \cos \left(\frac{\alpha \pi}{2}\right)+\left(\frac{\omega}{\omega_{c}}\right)^{2 \alpha}},
$$




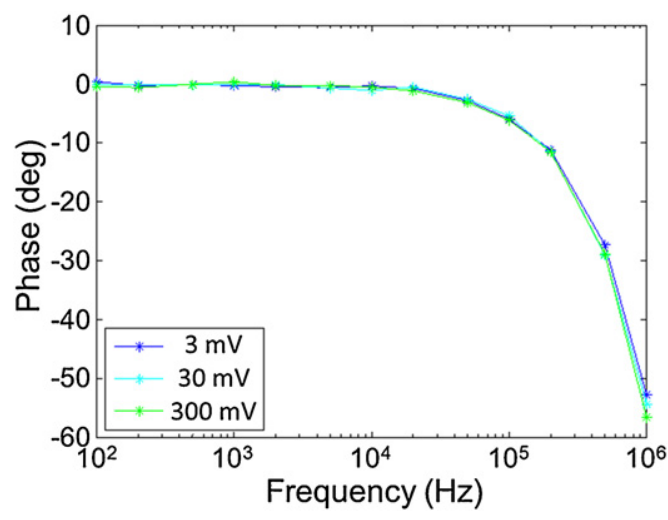

(a)

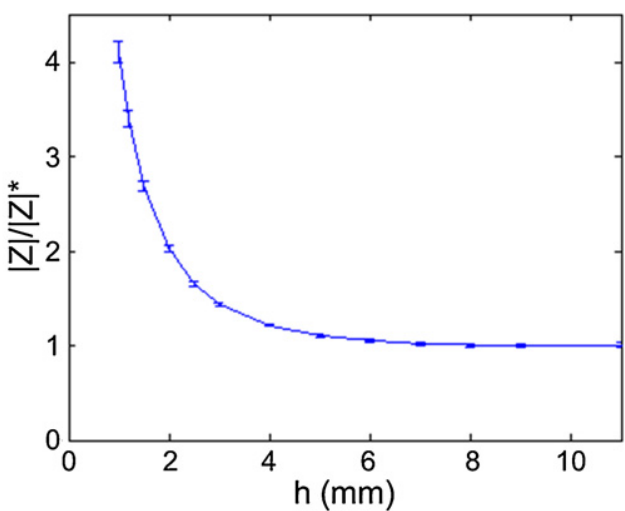

(b)

Figure 3. Results from the experimental characterization. (a) Plot of phase shift (degrees) versus frequency $(\mathrm{Hz})$ for varying differential voltage inputs into the instrumentation amplifier. Negligible phase shift is seen for frequencies up to $10 \mathrm{kHz}$ with increasingly negative phase shifting after that with increasing frequency. Phase shifts at $100 \mathrm{kHz}$ and $1 \mathrm{MHz}$ were measured at $-5.9^{\circ}$ and $-54.4^{\circ}$, respectively. (b) Plot of measured impedance magnitude $(|Z|)$ as a function of the bioimpedance probe depth $(h)$ for saline solutions of $0.9 \%, 0.125 \%$, and $0.01 \%$ concentrations at 10 frequency points from $100 \mathrm{~Hz}-100 \mathrm{kHz}$. All data points were normalized to the $|Z|$ at $h=11 \mathrm{~mm}\left(|Z|^{*}\right)$. Error bars in (b) are standard error $(n=30)$. The plot shows that as the bioimpedance probe approaches the bottom of the tissue chamber (decreasing $h$ ), the measured $|Z|$ increases in a consistent manner which is frequency and load independent.

where $R(\omega)$ is the real part of the bioimpedance measurement at frequency $\omega, R_{\infty}$ is the resistance at infinite frequency, $R_{0}$ is the resistance at zero frequency $(\mathrm{dc}), \omega_{c}$ is the characteristic frequency, and $\alpha$ is the dispersion coefficient. The extracellular resistance $\left(R_{\text {ext }}\right)$, intracellular resistance $\left(R_{\mathrm{int}}\right)$, and bulk membrane capacitance $\left(C_{m}\right)$ can be extracted from the fitted data using the following equations:

$$
\begin{aligned}
R_{\mathrm{ext}} & =R_{0} \\
R_{\mathrm{int}} & =\frac{R_{\infty} R_{0}}{R_{\infty}-R_{0}} \\
C_{m} & =\frac{1}{\omega_{c}\left(R_{\mathrm{int}}+R_{\mathrm{ext}}\right)} .
\end{aligned}
$$

\section{Results}

Findings for the experimental setup characterization, compression-dependent bioimpedance measurements, and the relationship between peak pressure and intracellular resistance are given below.

\subsection{Characterization of experimental setup}

Factors influencing the bioimpedance measurements were found to come from the IA circuitry, distance between the bioimpedance electrodes and the bottom of the tissue chamber, and stray capacitance within the experimental setup. Future versions of the bioimpedance circuitry will take these influences into account and condition the data in real-time. In this study, these influences were removed during post-processing.

Figure 3(a) shows the influence of frequency on the phase reading form the IA circuitry at differential input voltage signals of 3,30 , and $300 \mathrm{mV}_{\mathrm{RMS}}$. In an ideal instrumentation 


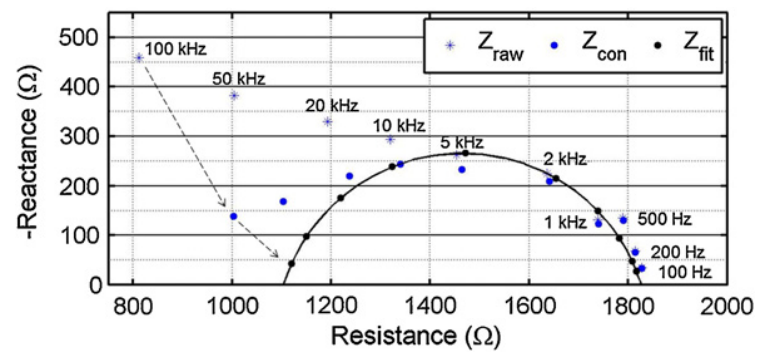

Figure 4. Nyquist plot of the absolute value of the imaginary part of the impedance (Reactance) against the real part of the impedance (Resistance) showing the development of the measured data from raw data $\left(Z_{\text {raw }}\right)$ to conditioned data $\left(Z_{\text {con }}\right)$ to final fitted data $\left(Z_{\text {fit }}\right)$ in the Cole-Cole model. The figure shows that the findings during the experimental characterization do not affect the low frequency data points (points further to the right in the figure) but have an increasingly important role in the measured raw data at high frequencies (points further to the left in the figure). The black dashed arrows show the change to the impedance at $100 \mathrm{kHz}$ from the raw data (blue star) to the conditioned data (blue solid circle) to the fitted data (black solid circle).

amplifier, there would be zero phase shift between the input and output signal. For the IA circuitry in this study, negligible phase shift is seen for frequencies up to $10 \mathrm{kHz}$ with an increasingly negative phase shift with increasing frequency beyond $10 \mathrm{kHz}$. Phase shifts at $100 \mathrm{kHz}$ and $1 \mathrm{MHz}$ were measured at $-5.9^{\circ}$ and $-54.4^{\circ}$, respectively. The phase shift is independent of the differential input voltage over a range of 3-300 mV and is therefore independent of the tissue sample impedance in the range of 50-5 k 2 . The gain for the IA circuitry is constant at $26 \mathrm{~dB}$ (gain of $20 \mathrm{~V} \mathrm{~V}^{-1}$ ) over the frequency range of interest $(100 \mathrm{~Hz}-$ $100 \mathrm{kHz}$ ).

Average measurements and standard error $(n=30)$ of $|Z|$ for frequencies between 100 $100 \mathrm{kHz}$ and $0.01 \%, 0.125 \%$, and $0.9 \%$ saline concentrations as a function of $h$ are shown in figure 3(b). The data for each saline concentration was normalized to $|Z|^{*}$ before averaging to show the influence of $h$ independent of the saline concentration. The figure shows that $h$ influences $|Z|$ in a consistent manner over a large range of saline concentrations and is consistent with finite element calculations performed to mirror this situation. The measured $|Z|$ increases as the $h$ decreases; however, the increase is independent of saline concentration, and therefore impedance, over the frequency range of $100 \mathrm{~Hz}-100 \mathrm{kHz}$. This result is consistent with results published by Tsai (Tsai 2000) which show that as a bioimpedance probe gets closer to a second material, in this case the bottom of the acrylic tissue chamber, the electric field becomes increasingly influenced by its presence resulting in a change in the apparent voltage drop measured by the bioimpedance probe. The average uncompressed tissue thickness was $9.2 \mathrm{~mm}$ (standard error $0.46 \mathrm{~mm}$ and $n=8$ ). At $80 \%$ compression, the tissue is $1.8 \mathrm{~mm}$ thick which results in an apparent increase in $|Z|$ of 2.29 times the $|Z|$ measured at an $h$ of $11 \mathrm{~mm}$. There was no influence of $h$ on the measured phase shift.

The average calculated stray capacitance, $C_{p}$, was $404 \mathrm{pF}$ (standard error of $40 \mathrm{pF}$ and $n=72$ ). As frequency increases, the electrical current is increasingly able to use this stray capacitance pathway to ground without being measured by the current-to-voltage converter circuitry which influences both the magnitude and phase of the measured bioimpedance measurement. In a Nyquist plot, this results in a characteristic tail developing on the left side of the impedance loci (see $Z_{\text {raw }}$ in figure 4) which can be compensated for using equation (1).

Both the current-to-voltage converter and the voltage-controlled current source circuits performed as expected. The current-to-voltage converter was able to measure $60 \mu \mathrm{A}_{\mathrm{RMS}}$ for 
impedances ranging from $50 \Omega-100 \mathrm{k} \Omega$ over the frequency range of interest $(100 \mathrm{~Hz}-$ $100 \mathrm{kHz}$ ) with negligible phase shift induced on the output signal compared to the input signal. The voltage-controlled current source was able to drive a $60 \mu \mathrm{A}_{\mathrm{RMS}}$ current signal with an output impedance of $950 \mathrm{k} \Omega$ through 100 . These loads are large enough to drive both the tissue and anticipated contact impedances over the frequency range of interest (Schwan 1968a).

The frequency-dependent phase shift from the IA, change in $|Z|$ due to $h$, and stray capacitance are each removed from the raw bioimpedance measurement $\left(Z_{\text {raw }}\right)$ as discussed above. After data conditioning is performed to account for these influences, the circuitry is capable of measuring tissue bioimpedance in the frequency range of $100 \mathrm{~Hz}-100 \mathrm{kHz}$ and a range of impedance loads $100 \Omega-5 \mathrm{k} \Omega$ as discussed below in section 3.2.

\subsection{Compression-dependent bioimpedance measurements}

Figure 4 shows a Nyquist plot of the $Z_{\text {raw }}$, conditioned measurement $\left(Z_{\text {con }}\right)$, and a least squares fit to a Cole-Cole model $\left(\mathrm{Z}_{\mathrm{fit}}\right)$ of a porcine spleen tissue sample. The measurement was taken at $50 \%$ compression and a height $(h)$ of $6.20 \mathrm{~mm}$, or a tissue with an uncompressed height of $12.40 \mathrm{~mm}$. $Z_{\text {raw }}$ is conditioned for the effects of the circuitry, $h$, and stray capacitance as described above in section 3.1. This conditioned data $\left(Z_{\text {con }}\right)$ is fit to a Cole-Cole model $\left(Z_{\text {fit }}\right)$ as described in section 2.4 and is plotted at each experimental frequency point (black solid circle) and as a continuous function (black line). The average root mean squared error (RMSE) for the fitting of $Z_{\text {con }}$ to the Cole-Cole model was $18.7 \Omega$ (standard error $=1.1 \Omega$ and $n=72$ ). The figure shows that the experimental characterization performed primarily affects the data at frequencies of $5 \mathrm{kHz}$ and higher (points further to the left in the figure). The black dashed arrows show the change to the impedance data point measured at $100 \mathrm{kHz}$ from the raw data (blue star) to the conditioned data (blue solid circle) to the fitted data (black solid circle).

Changes to the Cole-Cole fitted impedance loci for a sample porcine spleen tissue undergoing compression from $0-80 \%$ are shown together in a Nyquist plot in figure 5. At compression levels of up to $50 \%$ the loci diameters decrease slightly while the center of the loci shifts to the right along the real axis as compression is increased, correlating to an increase in the real component of impedance at each frequency. At compression levels greater than $50 \%$, the loci diameters collapse more dramatically while shifting back to the left along the real axis with increasing compression, indicating a decrease in interstitial resistance (from the shift in the loci to the left along the real axis) and an increase in intracellular resistance (from the decrease in loci diameter).

Extracted Cole-Cole parameters from the fitted impedance loci at each compression level for each trial $(n=8)$ were normalized to their uncompressed values and the averages and standard errors are shown in figure 6. The average uncompressed extracellular resistance, intracellular resistance, bulk membrane capacitance, and dispersion coefficient were $1230 \Omega$, $2220 \Omega, 8.9 \mathrm{pF}$, and 0.81 , respectively. The average dispersion coefficient $(\alpha)$ for all experiments and compression levels was 0.796 ( standard error $=0.0038$ and $n=72$ ). As $\alpha$ was not seen to change significantly from its uncompressed value, it is not plotted. The extracellular resistance $\left(R_{\text {ext }}\right)$ increases $11 \%$ up to $50 \%$ compression before dropping by $32 \%$ at $80 \%$ compression relative to its uncompressed value. The intracellular resistance $\left(R_{\text {int }}\right)$ increases $24 \%$ at $50 \%$ compression before continuing to increase to $107 \%$ at $80 \%$ compression relative to its uncompressed value. The bulk cellular membrane capacitance $\left(C_{\text {mem }}\right)$ decreases $13 \%$ at $50 \%$ compression before increasing $64 \%$ at $80 \%$ compression relative to its uncompressed value. 


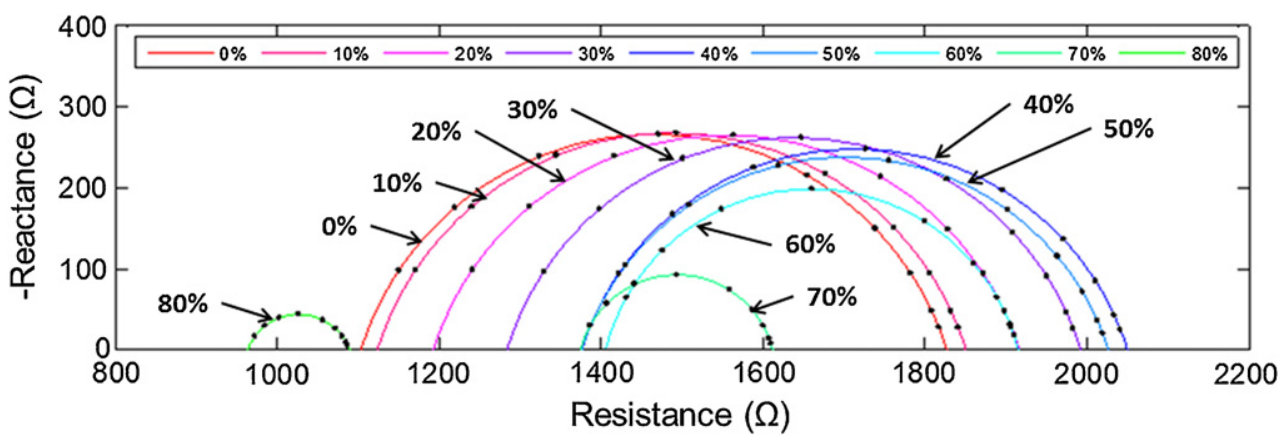

Figure 5. Nyquist plot of the absolute value of the imaginary part of the impedance (Reactance) against the real part of the impedance (Resistance) showing the development of the fitted impedance loci measured in porcine spleen tissue for 0-80\% tissue compression. At lower compression levels $(<50 \%)$, the figure shows the loci shifting to the right along the real axis while the loci diameter decreases slightly indicating an increase in intracellular and interstitial resistances. At higher compression levels ( $>50 \%$ ), the figure shows a more dramatic shift to the left along the real axis of the loci with a corresponding collapse of the loci diameter indicating a decrease in interstitial resistance (loci shifting to the left along the real axis)along with a continuing increase in intracellular resistance (loci diameter collapse).

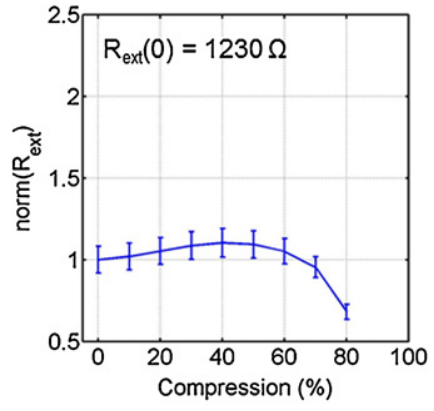

(a)

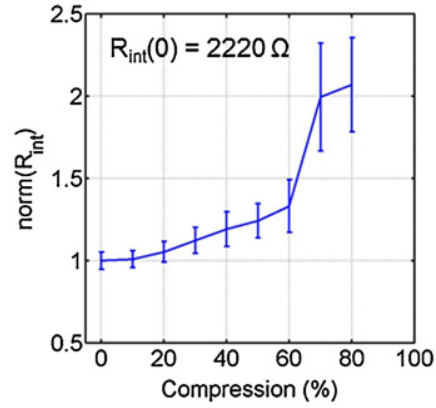

(b)

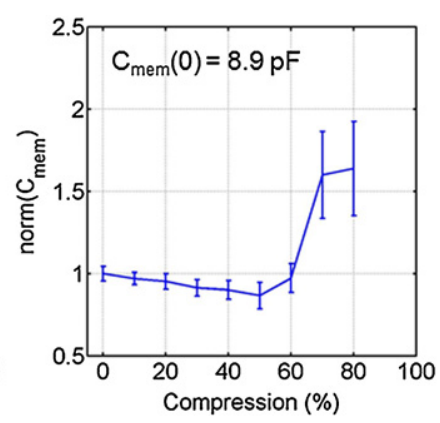

(c)

Figure 6. Summary of compression-dependent Cole-Cole parameter values normalized to their values at zero strain. (a) Plot of the interstitial resistance $\left(R_{\text {ext }}\right)$ as a function of percent compression. The plot shows an increase in $R_{\text {ext }}$ at low compression levels $(<50 \%)$ and a subsequent decrease in $R_{\text {ext }}$ as compression is increased beyond $50 \%$. (b) Plot of intracellular resistance $\left(R_{\text {int }}\right)$ as a function of percent compression. The plot shows a steady increase in $R_{\text {int }}$ with increasing compression resulting in a doubling of $R_{\text {int }}$ at $80 \%$ compression. (c) Plot of bulk tissue capacitance ( $\left.C_{\text {mem }}\right)$ as a function of percent compression. The plot shows a decrease in $C_{\mathrm{mem}}$ at low compression levels $(<50 \%)$ and a subsequent increase in $C_{\mathrm{mem}}$ as compression is increased beyond $50 \%$. Error bars are standard error $(n=8)$.

\subsection{Peak pressure versus intracellular resistance}

The experimentally measured stress relaxation during one test from $0-80 \%$ compression is shown in figure 7(a). As the porcine spleen is compressed, a large pressure spike develops in the tissue before relaxing to a steady-state pressure higher than the pressure value just prior to compression typical of visco- and poro-elastic materials. 


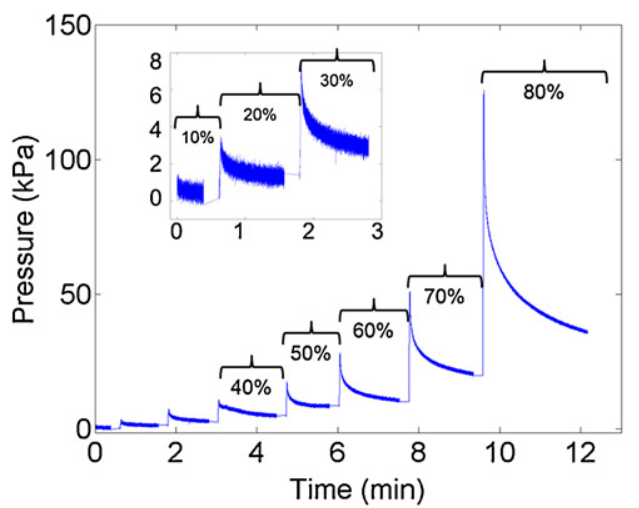

(a)

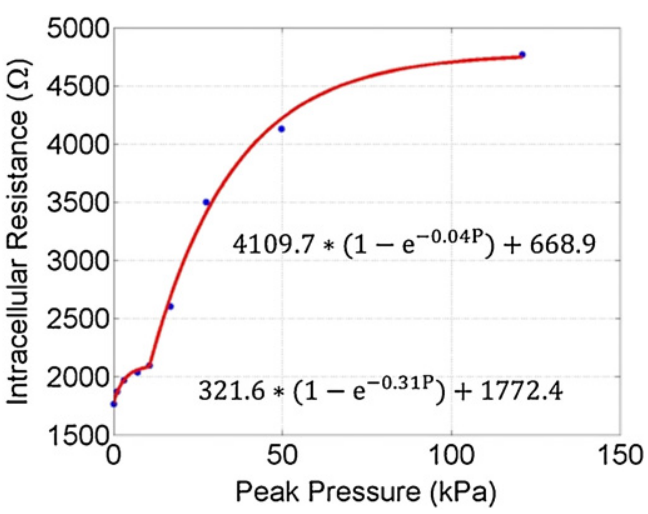

(b)

Figure 7. (a) Plot of stress relaxation as a function of time for a porcine spleen sample from 0-80\% compression (compression level identified in brackets). Inset shows zoomed in data for 10-30\% compression where the axes are in the same units as the parent graph. The plot shows a characteristic pressure spike as the tissue undergoes active compression followed by a relaxation in the pressure corresponding to the tissue relaxation as it is held at a constant compression level. (b) Plot of intracellular resistance as a function of peak pressure measured at each compression level for a porcine spleen tissue sample undergoing 0-80\% compression. Each data point (solid blue circle) represents a $10 \%$ increase in compression. The red lines are curve fits to the data showing two asymptotic plateaus for the intracellular resistance with increasing compression.

For $63 \%$ of the compression experiments ( 5 out of 8 ), plotting the peak pressure seen by the tissue against the measured intracellular resistance at each compression level reveals two distinct asymptotic curves as shown in figure 7(b). At compression levels up to $40 \%$, the $R_{\text {int }}$ asymptotically converges to a value of $2090 \Omega$ with increasing pressure. However, for higher compressions, the tissue experiences a second asymptotic increase in $R_{\text {int }}$, converging to a value of $4780 \Omega$. The average pressure at which the second asymptotic increase begins was measured to be $16.2 \mathrm{kPa}$ (standard error of $3.1 \mathrm{kPa}$ ).

\section{Discussions}

Bioimpedance data for porcine spleen tissue under compression show two phases of change. For strains less than $50 \%$, both $R_{\text {int }}$ and $R_{\text {ext }}$ increase slightly with increasing strain. With increasing compression, however, $R_{\text {ext }}$ decreases while $R_{\text {int }}$ increases dramatically. After initially decreasing with increasing strains up to $50 \%$, the bulk membrane capacitance, $C_{\text {mem }}$, increases rapidly with increasing strain. Certainly major mechanical changes are occurring at levels of high strain $(\varepsilon)$. When considering soft tissues undergoing compression, an updated Cole-Cole model must therefore be employed where the standard, constant resistor and capacitor values are replaced with strain-dependent values (i.e. $R_{\text {int }}(\varepsilon), R_{\text {ext }}(\varepsilon)$ and $C_{m}(\varepsilon)$ ).

Tissue bioimpedance is primarily determined by both the ionic concentration and the overall fluid fraction of the tissue (Grimnes and Martinsen 2008). Changes in bioimpedance, then, can occur based on changes to the electrolyte or fluid volume. Under tissue compression, pressure gradients developed within the tissue act to drive fluid out of the compressed space. It has been postulated previously that the fluid losses come from the extracellular tissue space; however, this research shows that losses from the intracellular space are also important. The interstitial space serves as a medium between the intracellular space and the outer tissue boundary. Fluid leaving the tissue during compression must be seen as potentially coming 
from both the intracellular and extracellular spaces. Understanding where this fluid comes from can play an important role in determining the underlying mechanisms for changes in tissue under compression. Bioimpedance can be used to decipher between these two changes and is therefore a promising technique to be used in studying compressive tissue injuries such as blunt trauma and traumatic brain injury. Additionally, considering the sensitivity of bioimpedance to the volume fraction of fluid in tissue, bioimpedance could theoretically be used to quantify hydration status in edematous patients such as in dialysis.

The intracellular impedance of porcine spleen tissue under compression experiences two asymptotic increases when plotted against its peak pressure seen during each level of induced strain (figure 7(b)). It is hypothesized that the asymptotic increase at low pressures may be due to intracellular fluid losses to the extracellular space through the cell membrane, possibly through aquaporin activation (Ishibashi et al 2009). The increase may also be in part due to the reshaping of cells from a spherical to oblong orientation. The reshaping of cells under compression has been documented previously (Demou 2010, Haudenschild et al 2009) and may lead to an apparent intracellular impedance change due to the shifting of field lines through cells. Additionally, the decreasing $C_{\text {mem }}$ could initially result from cell membranes contacting each other resulting in a serializing of the cell membranes and decreasing capacitance.

There are at least two distinct physiological phenomena that can explain the second asymptotic intracellular impedance increase observed at higher pressures. The first theory considers the case of cell rupture. Whereas at low increasing strains both interstitial and intracellular spaces see a decrease in conductance (or increased resistance), during increasing strains over $50 \%$ the conductance of the interstitial space begins to increase. The fluidic and ionic flux of the intracellular space during compression depends on losses to the outside as well as any gains/losses from the intracellular space. Cell membrane rupture results in intracellular contents from ruptured cells entering into the interstitial space creating a simultaneous drop in $R_{\text {ext }}$ while increasing $R_{\text {int }}$. The increase in $C_{\text {mem }}$ at higher strains would result from the loss of the cell membrane during cell rupture. This would be the equivalent of removing a series capacitor, creating a more parallel system and increasing $C_{\text {mem }}$.

The second theory involves the mass exudation of cellular material from the compressed space. In this scenario, cell membranes may not rupture, but increasing stress from compression eventually overcomes the proteinaceous adhesive bonds between the cell and other cells (cellcell adhesion) and between the cell and the extracellular matrix (cell-ECM adhesion) (Ingber 2006). Radial pressure gradients may force the cells out of the measurement space of the bioimpedance probe, leaving behind mostly fluid. At high compression levels, what would be left behind is a lower intracellular volume resulting in an increased $R_{\text {int }}$ and an increased $C_{\text {mem }}$ due to the loss of 'series'-ness in the cellular membranes.

These two theories, however, are not mutually exclusive. It is possible that both cell rupture and adhesion force breakdown may occur simultaneously. Further research is needed to determine which, if either, of these two theories is occurring at high tissue compression.

Looking at the impact these results have on electrosurgery, average impedance data calculated from the Cole-Cole model at a frequency of $500 \mathrm{kHz}$ is plotted in $10 \%$ strain increments from $0-80 \%$ in figure 8 . Typical tissue strains during electrosurgery can easily exceed $50 \%$. From $50-80 \%$ strains, a $31 \%$ loss in resistance is seen, preceded by a $15 \%$ increase in resistance. These finding indicate there is a level of compression that could be used during electrosurgery for minimizing the tissue impedance to electrosurgical current. Reducing tissue impedance would increase resistive heating in the tissue by increasing the current density at a specified power level.

While the experimental procedure used most closely resembled that of an electrosurgical procedure with relatively slow tissue compression, it is believed this methodology can be used 


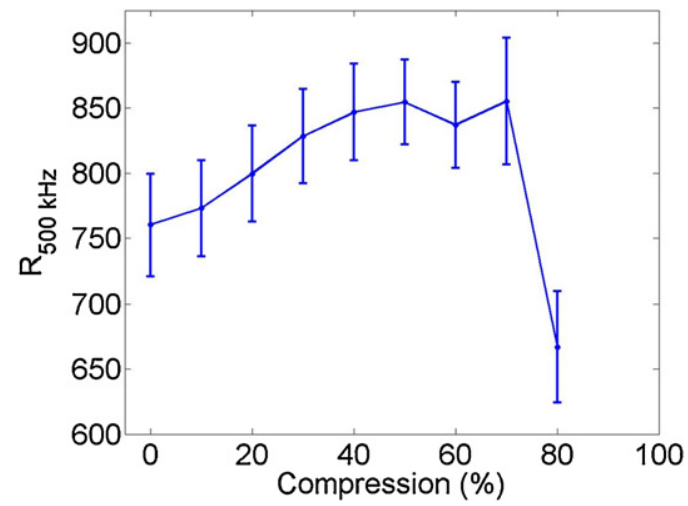

Figure 8. Plot of the measured tissue impedance $|Z|$ at standard electrosurgical frequency of 500 $\mathrm{kHz}$ as a function of tissue compression. Error bars are standard error $(n=8)$. The plot shows an initial increase in $|Z|$ through $50 \%$ compression with a subsequent decline in impedance as compression is increased further.

to analyze more complex phenomenon such as traumatic brain injury where tissue compression occurs on the order of milliseconds. Acquiring bioimpedance measurements over the entire cycle of tissue relaxation following a traumatic event may allow for analysis of how the cellular structure of tissue changes during as well as in the short and long term after such an event. However, care will need to be exercised in the analysis of real-time bioimpedance measurements using the tetrapolar technique due to the complex distribution of measurement sensitivity when using this approach. The appearance of negative sensitivity regions could give rise to erroneous conclusions as relative increases in impedance in these regions would yield measurements of decreasing impedance (Grimnes and Martinsen 2007). Under quasi-static conditions such as those employed in the current study, this impact is assumed to be averaged out by the tissue reaching a point of relaxation. However, in the dynamic case, this assumption cannot be made.

Analysis of the bioimpedance data could be aided by performing histology. However, initial histology performed on the compressed tissue samples in this study proved inconclusive in determining the cellular level changes in tissue at high compression. This is believed due to the natural relaxation of the tissue after the strain was removed. Additionally, while adequate steps were taken to prevent thermal effects from influencing the data, the addition of thermal monitoring to the experimental setup would allow for any thermal effects to be accounted for in the results.

\section{Conclusions}

This research quantified the impact that tissue compression has on bioimpedance measurements. At strains of $80 \%$ intracellular resistance increased $107 \%$, bulk membrane capacitance increased $64 \%$, and interstitial resistance decreased $32 \%$ relative to their uncompressed values. As opposed to previous assumptions that the loss of fluid during tissue compression is primarily from the extracellular space, these findings suggest that intracellular fluid losses are a critical component. Histology performed on the tissue samples was inconclusive as to the exact nature of change in the highly compressed tissue, but given the appearance of relatively unharmed cellular structures, cell rupture is believed to not be the major physiological phenomena responsible for the increase in $R_{\text {int }}$. However, an 
exudation of cellular material after adhesion forces are broken at higher pressures may be satisfactory to explain the bioimpedance changes seen in the tissue. Performing histology on tissue samples while still under compression could aid in determining the underlying mechanism of bioimpedance change seen in this data and is a future research direction. The ability for bioimpedance measurements to distinguish intracellular and extracellular events in tissue during compression may also have uses in quantifying the changes in the fluid phase of poroelastic materials and in understanding injury mechanisms in cases such as traumatic brain injury.

\section{References}

Abramowitch S D 2004 An improved method to analyze the stress relaxation of ligaments following a finite ramp time based on the quasi-linear viscoelastic theory J. Biomech. Eng. 12692

Ackmann J J 1993 Complex bioelectric impedance measurement system for the frequency range from $5 \mathrm{~Hz}$ to $1 \mathrm{MHz}$ Ann. Biomed. Eng. 21 135-46

Ayllon D, Seoane F and Gil-Pita R 2009 Cole equation and parameter estimation from electrical bioimpedance spectroscopy measurements-a comparative study Conf. Proc. IEEE Eng. Med. Biol. Soc. 2009 p 3779

Berry G P, Bamber J C, Mortimer P S, Bush N L, Miller N R and Barbone P E 2008 The spatio-temporal strain response of oedematous and nonoedematous tissue to sustained compression in vivo Ultrasound Med. Biol. 34 617-29

Bolton M P, Ward L C, Khan A, Campbell I, Nightingale P, Dewit O and Elia M 1998 Sources of error in bioimpedance spectroscopy Physiol. Meas. 19235

Bouty E 1884 Sur la conductibilité électrique des dissolutions salines très étendues J. Phys. 3 325-55

Bowdler A J 2002 The Complete Spleen: Structure, Function, and Clinical Disorders (Totowa, NJ: Humana Press)

Brown B H, Wilson A J and Bertemes-Filho P 2000 Bipolar and tetrapolar transfer impedance measurements from volume conductor Electron. Lett. 36 2060-2

Buendía R, Seoane F and Gil-Pita R 2010 A novel approach for removing the hook effect artefact from electrical bioimpedance spectroscopy measurements J. Phys.: Conf. Ser. 224012126

Cole K S and Cole R H 1941 Dispersion and absorption in dielectrics: I. Alternating current characteristics J. Chem. Phys. $9341-51$

Demou Z N 2010 Gene expression profiles in 3D tumor analogs indicate compressive strain differentially enhances metastatic potential Ann. Biomed. Eng. 38 3509-20

Dodde R E, Miller S F, Geiger J D and Shih A J 2008 Thermal-electric finite element analysis and experimental validation of bipolar electrosurgical cautery J. Manuf. Sci. Eng. 130021015

Eaton J 2005 Kinetics and mechanics of injury Principles and Practice of Trauma Nursing ed R A O'Shea (Edinburgh: Elsevier) pp 15-35

Fung Y C 1993 Biomechanics: Mechanical Properties of Living Tissues (New York: Springer-Verlag)

González-Correa C A 2005 Electrical bioimpedance readings increase with higher pressure applied to the measuring probe Physiol. Meas. $26 \mathrm{~S} 39$

Grimnes S and Martinsen $\varnothing \mathrm{G} 2007$ Sources of error in tetrapolar impedance measurements on biomaterials and other ionic conductors J. Phys. D: Appl. Phys. 40 9-14

Grimnes S and Martinsen Ø G 2008 Bioimpedance and Bioelectricity Basics (London: Academic)

Haudenschild A, Hsieh A, Kapila S and Lotz J 2009 Pressure and distortion regulate human mesenchymal stem cell gene expression Ann. Biomed. Eng. 37 492-502

Ingber D E 2006 Cellular mechanotransduction: putting all the pieces together again FASEB J. 20811

Ishibashi K, Hara S and Kondo S 2009 Aquaporin water channels in mammals Clin. Exp. Nephrol. 13 107-17

Jakosky J J 1961 Exploration Geophysics (Newport Beach, CA: Trija Pub. Co.)

Keshtkar A 2008 The effect of applied pressure on the electrical impedance of the bladder tissue using small and large probes J. Med. Eng. Technol. 32 505-11

Kottam A 2004 Electric field penetration depth of myocardial surface catheters and the measurement of myocardial resistivity Biomed. Sci. Instrum. 449 155-60

Littwitz C, Ragheb T and Geddes L 1990 Cell constant of the tetrapolar conductivity cell Med. Biol. Eng. Comput. 28 587-90

McEwan A, Cusick G and Holder D S 2007 A review of errors in multi-frequency EIT instrumentation Phys. Meas. 28 S197-215 
Mow V C, Kuei S C, Lai W M and Armstrong C G 1980 Biphasic creep and stress relaxation of articular cartilage in compression-theory and experiments J. Biomech. Eng. 102 73-84

Pena A, Bolton M D and Pickard J D 1998 Cellular poroelasticity: a theoretical model for soft tissue mechanics Poromechanics 475-80

Rosen J, Brown J D, De S, Sinanan M and Hannaford B 2008 Biomechanical properties of abdominal organs in vivo and postmortem under compression loads J. Biomech. Eng. 130021020

Rylander C G, Stumpp O F, Milner T E, Kemp N J, Mendenhall J M, Diller K R and Welch A J 2006 Dehydration mechanism of optical clearing in tissue J. Biomed. Opt. 11041117

Scharfetter H 1998 A model of artefacts produced by stray capacitance during whole body or segmental bioimpedance spectroscopy Physiol. Meas. 19247

Schwan H 1968a Electrode polarization impedance and measurements in biological materials Ann. N. Y. Acad. Sci. 148 191-209

Schwan H P 1968b Four-electrode null techniques for impedance measurement with high resolution Rev. Sci. Instrum. 39 481-5

Schwan H P and Foster K R 1980 RF-field interactions with biological systems: electrical properties and biophysical mechanisms Proc. IEEE $\mathbf{6 8} 104-13$

Steendijk P, Baan J, Mur G and Van Der Velde E T 1993 The four-electrode resistivity technique in anisotropic media: theoretical analysis and application on myocardial tissue in vivo IEEE Trans. Biomed. Eng. 40 1138-48

Tamura A, Omori K, Miki K, Lee J B, Yang K H and King A I 2002 Mechanical characterization of porcine abdominal organs Stapp Car Crash J. 4655

Tsai J Z 2000 Dependence of apparent resistance of four-electrode probes on insertion depth IEEE Trans. Biomed. Eng. 47 41-8

Wojslaw C F and Moustakas E A 1986 Operational Amplifiers: the Devices and Their Applications (New York: Wiley) 